\title{
Letter to the Editor re: In Vivo Whole Animal Body Imaging Reveals Colonization of Chlamydia muridarum to the Lower Genital Tract at Early Stages of Infection
}

\author{
Andrew P. Craig, David P. Wilson \\ The Kirby Institute, UNSW Australia, Sydney, NSW, 2052, Australia
}

$\mathrm{T}^{\mathrm{T}}$ here is limited knowledge of the processes involved in chlamydial ascension from the lower to the upper genital tract. We congratulate Gupta and colleagues for their imaging study of infection of the mouse genital tract with Chlamydia muridarum, which addressed this topic [1]. Among other results, they find that the movement of spherobeads from the lower to the upper genital tract was similar to that of the chlamydiae. They see this as evidence against a contribution of fluid movement to chlamydial colonization. However, we would expect spherobead movement to be predominantly due to fluid movement, and if chlamydiae movement is similar, we could attribute that to fluid movement as well.

Our simple modeling of chlamydia ascension with the assumption of the existence of a diffusion-type process, as might be expected if ascension was due to fluid movement, is consistent with the data from Gupta et al. (results not shown). The available evidence is not conclusive on the mechanism for ascension, but our interpretation of the data is that it is likely purely fluid movement. Polymorphonuclear leukocytes have been observed to detach infected cells from the epithelium [2], and it may be that both free chlamydiae and infected cells ascend the genital tract due to fluid movement.

As noted by Gupta et al., there have been mathematical modeling studies that incorporate spatial aspects of genital tract chlamydial infection [3-5]. However, these models have been difficult to parameterize due to the lack of spatial data. We anticipate that joint imaging and modeling studies, in which calibration curves allow the quantification of chlamydiae from images, could further elucidate the processes underlying chlamydial ascension.

Conflict of Interest. The authors declare that they have no conflict of interest.

\section{References}

1. Gupta R, Wali S, Yu J-J et al (2014) In vivo whole animal body imaging reveals colonization of Chlamydia muridarum to the lower genital tract at early stages of infection. Mol Imaging Biol. doi:10.1007/s11307-014$0732-5$

2. Rank RG, Judy Whittimore J, Bowlin AK et al (2011) In vivo ultrastructural analysis of the intimate relationship between polymorphonuclear leukocytes and the chlamydial developmental cycle. Infect Immun 79:3291-3301

3. Mallet D, Heymer K, Wilson DP (2007) A novel cellular automatapartial differential equation model for understanding chlamydial infection and ascension of the female genital tract. Proc Appl Math Mech 7:2120001-2120002

4. Mallet DG, Heymer KJ, Rank RG, Wilson DP (2009) Chlamydial infection and spatial ascension of the female genital tract: a novel hybrid cellular automata and continuum mathematical model. FEMS Immunol Med Microbiol 57:173-182

5. Mallet DG, Bagher-Oskouei M, Farr AC et al (2013) A mathematical model of Chlamydial infection incorporating movement of Chlamydial particles. Bull Math Biol 75:2257-2270 